\title{
PUBLICAÇÕES, VÍdEOS, PROGRAMAS DE RÁDIO E PÁGINAS NA INTERNET
}

A divulgação das iniciativas inovadoras empreendidas por governos subnacionais constitui um dos objetivos principais do Programa Gestão Pública e Cidadania e envolve, além da difusão de um banco de dados com mais de 7.000 experiências (disponível na página da internet http://inovando.fgvsp.br), a realização de pesquisas e estudos que contribuam, por exemplo, para a identificação de elementos inovadores e de eventuais obstáculos à introdução de mudanças na administração pública subnacional no Brasil. Nas próximas páginas disponibilizamos uma lista de nossas publicações.

O Programa Gestão Pública, além das publicações, vem produzindo desde 1998 vídeos como forma de disseminar experiências e práticas inovadoras, identificadas e analisadas ao longo dos seus ciclos de premiação. Em 2003, o Programa Gestão Pública e Cidadania iniciou uma nova etapa na disseminação de práticas inovadoras por meio de vídeos com o projeto Práticas Públicas em Construção. Essa iniciativa - desenvolvida com o apoio do BNDES, e em parceria com a TV VIVA - Centro de Cultura Luis Freire (Olinda-PE) - consiste em uma nova série de vídeos sobre temas relacionados à gestão local, a partir de algumas experiências registradas pelo Programa. O objetivo é facilitar o acesso a informação, estimulando o debate sobre possibilidades e caminhos de ação.

Em parceria com o CRIAR BRASIL, organização não governamental sediada do Rio de Janeiro, o Programa vem produzindo, desde 1999, programas de rádio sobre projetos inovadores e também sobre questões ligadas à cidadania. A série Buscando Soluções e a série de rádio-novelas Supermercado Mesa Pronta são distribuídas gratuitamente para mais de 400 rádios comunitárias, educativas e comerciais espalhadas pelo país.

Com o objetivo de ampliar os canais de disseminação, o Programa Gestão Pública e Cidadania também mantém duas páginas na internet. 


\section{PUBLICAÇÕES}

\section{CADERNOS GESTÃO PÚBLICA E CIDADANIA}

Volume 1: A Administração Pública Brasileira Inovando a Forma de Governar: apresentação dos 629 programas inscritos no Ciclo de Premiação 1996 / Ricardo Ernesto Vasquez Beltrão (org.), 1997.

Volume 2: Criação do Capital Social: o caso da Asmare / Pedro Jacobi e Marco Antonio Carvalho Teixeira, 1997.

Volume 3: Desafio e Inovação em Políticas Públicas: programas para crianças e adolescentes em situação de risco / Laura Veiga, Bruno Lazarotti Diniz Costa e Carla Bronzo Ladeira Carneiro, 1997.

Volume 4: Governo Local e Novas Formas de Provisão e Gestão de Serviços Públicos no Brasil / Marta Ferreira Santos Farah, 1997.

Volume 5: Gestão Pública em Busca de Cidadania: experiências de inovação em Salvador / José Antônio Gomes de Pinho, Mercejane Santana e Sonia Cerqueira, 1997.

Volume 6: Estudo Comparativo de Resultados Alcançados por Programas na Área de Saúde / Humberto Marques Filho, 1997.

Volume 7: Gestão Pública e Cidadania: metodologias participativas em ação / Fernando Guilherme Tenório e Jacob E. Rozenberg, 1997

Volume 8: A Administração Pública Brasileira Inovando a Forma de Governar: apresentação dos 297 programas inscritos no Ciclo de Premiação 1997 / Ricardo Ernesto Vasquez Beltrão (org.), 1997.

Volume 9: Avaliação da Importância de Atributos de Projetos de Desenvolvimento Inovadores / Luis Roque Klering, Roberto Costa Fachin e Zilá Mesquita, 1997.

Volume 10: Relatório sobre as Experiências Semifinalistas do Programa Gestão Pública e Cidadania - Ciclo de Premiação 1997 / Fernando Guilherme Tenório e Augusto P. G. Cunha, 1997. 
Volume 11: PROVE: uma experiência de implantação de microempresas agroindustriais / Humberto Marques Filho e Dilma Maria Guedes, 1998.

Volume 12: A Administração Pública Brasileira Inovando a Forma de Governar: apresentação dos 631 programas inscritos no Ciclo de Premiação 1998 / Ricardo Ernesto Vasquez Beltrão, Carlos Eduardo Evangelisti Mauro e Patrícia Laczynski (org.), 1999.

Volume 13: Programas para Crianças e Adolescentes em Situação de Risco: a complexidade do objeto e a dimensão institucional / Bruno Lazarotti Diniz Costa, Carla Bronzo Ladeira Carneiro e Carlos Aurélio Pimenta de Faria, 1999.

Volume 14: Uma Releitura dos Programas Selecionados nos Ciclos de Premiação de 1996 e 1997 / Humberto Marques Filho e André Luiz Felisberto, 1999.

Volume 15: A Sociedade é Protagonista na Relação com o Estado? / Fernando Guilherme Tenório e Gylcilene Ribeiro Storino, 2000.

Volume 16: A Administração Pública Brasileira Inovando a Forma de Governar: apresentação dos 888 programas inscritos no Ciclo de Premiação 1999 - Patrícia Laczinski, Sabrina Addison Baracchini e Ricardo Ernesto Vasquez Beltrão (org.), 2000.

Volume 17: The Rights Approach to Subnational Government: the experience of the public management and citizenship program - Peter Spink, 2000.

Volume 18: Parcerias, Novos Arranjos Institucionais e Políticas Públicas Locais - Marta Ferreira Santos Farah, 2000.

Volume 19: A Administração Pública Brasileira Inovando a Forma de Governar: apresentação dos 946 programas inscritos no Ciclo de Premiação 2000 - Marta Ferreira Santos Farah, Patrícia Laczynski, Paulo Jábali Júnior e Odélio Rodarte Arouca Filho (orgs.), 2000.

Volume 20: O Governo Municipal no Brasil: construindo uma nova agenda política na década de 90 - José Antônio Gomes de Pinho e Mercejane Wanderley Santana, 2000.

Volume 21: A Administração Pública Brasileira Inovando a Forma de Governar: apresentação dos 727 programas inscritos no Ciclo de Premiação 2001 - Peter Spink, Patrícia Laczynski e Francine Lemos Arouca (orgs.), 2002. 
Volume 22: A Administração Pública Brasileira Inovando a Forma de Governar: apresentação dos 981 programas inscritos no Ciclo de Premiação 2002 - Peter Spink, Francine Lemos Arouca e Marco Antônio Carvalho Teixeira (orgs.), 2003.

Volume 23: Informação, transparência e cidadania - o controle da execução orçamentária pelo cidadão. Peter Spink, 2003

Volume 24: Projeto Saúde e Alegria: um ensaio crítico. Mônica Mazzer Barroso, 2003.

Volume 25: The Brazilian Public Management and Citizenship Program: an Overview. Marta Farah e Peter Spink, 2003.

Volume 26: Estudo da Continuidade dos Projetos Educacionais do Município de Icapuí. Gabriela Lotta e Rafael Martins, 2003.

Volume 27: Análise da atuação institucional do Poder Judiciário e de agentes afins. Daniel Strauss, 2003.

Volume 28: Exclusão social e políticas públicas:algumas reflexões a partir das experiências descritas no Programa Gestão Pública e Cidadania. Carla Bronzo Ladeira Carneiro e Bruno Lazarotti Diniz Costa, 2003.

Volume 29: Tributação e política fiscal no nível municipal um estudo de caso: o programa de fortalecimento financeiro do Município de Vitória da Conquista. Fernanda Telles de Lima e Sílvia Craveiro, 2003.

Volume 30: Análise dos Projetos da Área Legislativa inscritos no Programa Gestão Pública e Cidadania. Fernando Machado, 2003.

Volume 31: Mitos e Realidades sobre a Inclusão Social: Participação Cidadã e Desenvolvimento Local. Silvio Caccia Bava, 2003.

Volume 32: Innovations in Government from around the World. Peter Spink e Jacqueline Brigagão, 2003.

Volume 33: Inovações de Governos Locais - Ciclo de Premiação 2003. Peter Spink, Lilia Asuca Sumiya, Marco Antonio Teixeira, Paula Pedroti (orgs.) 2003.

Volume 34: Disseminação de Experiências de Gestão Pública - O Caso do Programa de Renda Mínima no Brasil (1991-1997). Verônika Paulics 2004. 
Volume 35: Inovação na Gestão Municipal no Brasil: A Voz dos Gestores Municipais. José Antonio Gomes de Pinho 2004.

Volume 36: Políticas Sociais e Descentralização na América Latina I. Dagmar Raczynski, Claudia Serrano, Juan Fernández, Maria Adelaida Farah Q.,Edelmira Pérez C. , César Ortiz G. 2004.

Volume 37: Políticas Sociais e Descentralização na América Latina II. Cabrero E, Joseph J, Ricci J.L. 2004.

Volume 38: A Influência do FUNDEF no processo de municipalização do Ensino Fundamental no Estado de São Paulo. Patrícia Lacynsky 2004.

Volume 39: Agenda internacional de gestão urbana e o caso do Programa Ribeira Azul. Antonio Sérgio Araujo Fernandes 2004.

Volume 40: Negociação Política e interação Executivo Legislativo: A gestão Maluf no governo da cidade de São Paulo ( 1993 - 1996). Marco Antonio Teixeira. 2004

Volume 41: Innovations in Government from around the World.Peter Spink, Jacqueline Brigagão. 2004 


\section{OUTRAS PUBLICAÇÕES}

20 Experiências de Gestão Pública e Cidadania. SPINK, Peter e CLEMENTE, Roberta (org.). Estudo das 20 experiências finalistas do ciclo de premiação 1996.

20 Experiências de Gestão Pública e Cidadania. FUJIWARA, Luis Mario, ALESSIO, Nelson Luiz Nouvel e FARAH, Marta Ferreira Santos (org.). Estudo das 20 experiências finalistas do ciclo de premiação 1997.

20 Experiências de Gestão Pública e Cidadania - 1998. FUJIWARA, Luis Mario, ALESSIO, Nelson Luiz Nouvel e FARAH, Marta Ferreira Santos (org.). Estudo das 20 experiências finalistas do ciclo de premiação 1998.

Novas Experiências de Gestão Pública e Cidadania. FARAH, Marta Ferreira Santos e BARBOZA, Hélio Batista (orgs.). Estudo das 20 experiências finalistas do ciclo de premiação 1999.

20 Experiências de Gestão Pública e Cidadania - Ciclo de Premiação 2000. FARAH, Marta Ferreira Santos e BARBOZA, Hélio Batista (orgs.). Estudo das 20 experiências finalistas do ciclo de premiação 2000 .

20 Experiências de Gestão Pública e Cidadania - Ciclo de Premiação 2001. SPINK, PETER e BARBOZA, Hélio Batista (orgs.). Estudo das 20 experiências finalistas do ciclo de premiação 2001.

20 Experiências de Gestão Pública e Cidadania - Ciclo de Premiação 2002. SPINK, PETER; LOTTA, Gabriela S.; BARBOZA, Hélio Batista; TEIXEIRA, Marco Antonio C.; PINTO, Verena (orgs.). Estudo das 20 experiências finalistas do ciclo de premiação 2002.

Descobrindo o Brasil Cidadão - 1999. FARAH, Marta Ferreira Santos, SOARES, Ana Paula Macedo, BARBOZA, Hélio Batista Barboza e FUJIWARA, Luis Mário. Estudo resumido das 20 experiências finalistas do ciclo de premiação 1999.

Histórias de um Brasil que Funciona. - 2000. FARAH, Marta Ferreira Santos, BARBOZA, Hélio Batista Barboza e FUJIWARA, Luís Mário. Estudo resumido das 20 experiências finalistas do ciclo de premiação 2000. 
Histórias de um Brasil que Funciona - 2001. SPINK, Peter e BARBOZA, Hélio Batista Barboza. Estudo resumido das 20 experiências finalistas do ciclo de premiação 2001.

Histórias de um Brasil que Funciona - 2002. SPINK, Peter e BARBOZA, Hélio Batista Barboza. Estudo resumido das 20 experiências finalistas do ciclo de premiação 2002.

Histórias de um Brasil que Funciona - 2003. SPINK, Peter e BARBOZA, Hélio Batista Barboza. Estudo resumido das 20 experiências finalistas do ciclo de premiação 2003.

Innovative Stories: Indigenous experiences in Brazil, Chile and México - 2003. Programa Gestão Pública e Cidadania. Livro temático sobre os indígenas

\section{PUBLICAÇÕES PRÁTICAS PÚBLICAS E POBREZA}

Estratégias Locais para Redução da Pobreza: Construindo a Cidadania. CAMAROTTI, Ilka e SPINK, Peter (orgs.). Relatório final das oficinas temáticas e itinerantes realizadas em 1998 e 1999 pelo Projeto Práticas Públicas e Pobreza.

Parcerias e Pobreza: Soluções Locais na Construção de Relações Socioeconômicas. CAMAROTTI, Ilka e SPINK, Peter (orgs). Essa publicação é resultado de um trabalho de pesquisa e avaliação de projetos de redução da pobreza, realizado pelo Projeto Práticas Públicas e Pobreza com o apoio do Instituto do Banco Mundial em Washington e do escritório do Banco Mundial em Brasília. Os estudos apresentados nesse livro têm como enfoque as diferentes alianças ou parcerias desenvolvidas entre esferas de governo, organizações não-governamentais e comunitárias e empresas privadas. São algumas das muitas experiências que sinalizam contribuições possíveis em face da pobreza e da exclusão social.

Parcerias e Pobreza: Soluções Locais na Implementação de Políticas Sociais. CAMAROTTI, Ilka e SPINK, Peter (orgs.). As experiências analisadas nessa publicação chamam a atenção para as possibilidades e, também para as dificuldades e incertezas inerentes à produção de um conhecimento prático que pode servir de instrumento para a ação. Este livro foi realizado pelo Projeto Práticas Públicas e Pobreza com o apoio do Instituto do Banco Mundial em Washington e do escritório do Banco Mundial em Brasília. 
Redução da Pobreza e Dinâmicas Locais. CAMAROTTI, Ilka e SPINK, Peter (orgs). Os estudos apresentados neste livro mostram que é possível transformar a realidade e que trabalhar em conjunto, mobilizar, conscientizar, valorizar as potencialidades locais e compartilhar tarefas e responsabilidades são elementos essenciais para o êxito de projetos que visam reduzir a pobreza e as desigualdades. Esta publicação foi financiada pelo escritório do Banco Mundial em Brasília.

Estratégias Locais para Redução da Pobreza: Construindo a Cidadania. CAMAROTTI, Ilka e SPINK, Peter (orgs.). Apresentação de experiências, debates, aprendizagens e consensos de oficinas temáticas e itinerantes promovidas em 1998 e 1999 pelo Projeto Práticas Públicas e Pobreza. $2^{\circ}$ Edição, 2003.

O Que as Empresas Podem Fazer pela Erradicação da Pobreza. CAMAROTTI, Ilka e SPINK, Peter. Análise do conceito de pobreza, dados contextualizados sobre a magnitude do problema no Brasil e os caminhos possíveis de ação e, também de estímulo para a participação do setor empresarial no processo de mudança da sociedade brasileira, numa perspectiva de desenvolvimento econômico com justiça social. Realizado em parceria com o Instituto Ethos de Responsabilidade Social Empresarial.

Governo Local e Desigualdades de Gênero. CAMAROTTI, Ilka e SPINK, Peter (orgs.). Apresenta os estudos de cinco programas e projetos bem-sucedidos que estão sendo executados em diferentes regiões do Brasil. Cada experiência foi analisada especificamente em termos do seu impacto nas questões de gênero e sua sensibilidade a esta temática. São Paulo: Annablume, 2003. Edição bilíngüe (português e espanhol). Este livro contou com o apoio financeiro da Fundação Hewlett e da Agência Sueca de Cooperação Internacional para o Desenvolvimento (Asdi).

Estratégias para Redução da Pobreza: Caminhos de Ação. CAMAROTTI, Ilka e SPINK, Peter. Texto elaborado para o fórum de discussão "práticas locais na redução da pobreza: governança e sustentabilidade", realizado em Olinda (Pernambuco), dias 18 e 19 de março de 2004. Este encontro foi promovido pelo Programa Gestão Pública e Cidadania, pela Universidade de Westminster de Londres e pela Universidade Federal de Pernambuco, com o apoio financeiro da Fundação Hewlett, da Fundação Ford e do Programa UN-Habitat. 


\section{VÍDEOS}

\section{Novos Caminhos para uma Gestão Pública com Cidadania. (1998)}

Apresenta o Programa e estuda as tendências de inovação observadas nos governos subnacionais através de diversos exemplos de experiências dos ciclos de premiação de 1996 e 1997.

Série Brava Gente Brasileira. (1999)

Panorama de programas em diferentes áreas temáticas, produzido pela TV Futura.

Apresentando o Programa Gestão Pública e Cidadania. (2001)

Apresentação geral do Programa e seus objetivos.

Finalistas do Ciclo de 1999. (2001)

Panorama dos programas finalistas do ano de 1999.

Construindo Cidadania. (2001)

Introduz o Programa e os seguintes temas: a administração pública na ótica dos direitos humanos, cidadania política e cidadania social, tendências observadas nos programas inscritos, exemplos de projetos selecionados entre os finalistas de 2000 e, por último os depoimentos com a questão "onde estamos na conquista da cidadania dentro do espaço público?".

Combate à Pobreza na Gestão Pública. (2001)

Apresenta o quadro de pobreza no Brasil, análise do conceito de pobreza e a importância das parcerias e alianças; além disso, mostra os programas de combate à pobreza e formação de parcerias reunidos no banco de dados e alguns exemplos de projetos selecionados entre os finalistas de 2000; por fim, apresentam-se depoimentos referentes ao tema "As experiências de parceria alteram a responsabilidade de governos na prestação de serviços?"

Parcerias, Pobreza e Cidadania. (1999)

Apresenta três iniciativas estudadas pelo Projeto Práticas Públicas e Pobrezas: APAEB de Valente (BA), Programa SISAR (CE), e o ASMARE, de Belo Horizonte (MG). 


\section{Série Práticas Públicas em Construção.}

O objetivo desta série é facilitar o acesso a informações sobre possibilidades de ação e estimular o debate em torno de abordagens alternativas. Os sete primeiros vídeos da série já estão disponíveis:

1) Violência contra a mulher - sem medo de meter a colher. (2003)

Mostra um conjunto de ações desenvolvidas no município de Camaragibe (PE), destinadas às mulheres em situação de risco social ou vitimadas por qualquer tipo de violência.

\section{2) Agricultura Familiar - tradição que tem futuro. (2003)}

Retrata três iniciativas que buscam o desenvolvimento socioeconômico local por meio da agricultura familiar e comunitária. Ilustram este tema o Programa de Verticalização da Produção Agrícola do Pantanal (MS), o Fundo Municipal de Aval de Poço Verde (SE) e o Projeto Castanha (AP).

3) Consórcios Municipais - a descoberta do conjunto. (2003)

Ilustra as soluções advindas do consorciamento de 15 municípios no Maranhão (Consórcio Intermunicipal de Produção e Abastecimento, CINPRA) e de três municípios catarinenses, São Bento do Sul, Rio Negrinho e Campo Alegre (Consórcio Quiriri), que reuniram esforços na preservação ambiental.

4) Gestão Participativa - uma proposta, vários caminhos. (2003)

Apresenta o Programa Sobral Criança (CE) e o Orçamento Participativo de Porto Alegre (RS), duas experiências que ilustram alternativas possíveis para a implementação de formas de participação da sociedade na administração pública.

5) Cultura - cidadania e inclusão social. (2004)

Retrata duas iniciativas que privilegiam a promoção da cultura como instrumento de emancipação e inclusão social: o projeto Barracões Culturais da Cidadania, do município de Itapecerica da Serra (SP) e as ações da Fundação Carlos Gomes, no estado do Pará.

6) Saúde - prevenir é o mellhor remédio. (2004) 
Mostra um conjunto de ações desenvolvidas em Londrina (PR), que vem aperfeiçoando a implantação do Programa Saúde da Família nas zonas rural e urbana do município.

7) Educação - o direito de aprender. (2004)

Apresenta duas iniciativas que consideram as especificidades da realidade local na promoção da educação: a Escola Família Agroindustrial de Turmalina (MG) e o Programa de Erradicação do Trabalho Infantil (PETI) de Três Lagoas (MS).

\section{PROGRAMAS DE RÁDIO}

\section{Buscando Soluções - O Brasil Trocando Idéias (1999)}

As iniciativas governamentais de todo o Brasil premiadas em 1997 e 1998 pelo Programa Gestão Pública e Cidadania foram traduzidas para a linguagem do rádio por meio dos programas no estilo documentário desta série. Os projetos deram origem a 20 programas, com duração de 10 minutos cada.

\section{Buscando Soluções - Sempre Uma Boa Idéia no Ar (2001)}

Essa série de programas, que privilegiou as iniciativas premiadas em 1999 pelo Gestão Pública e Cidadania, tem por objetivo ampliar para todo o Brasil a voz dos gestores públicos e população beneficiária de programas e projetos inovadores em áreas como saúde, educação, desenvolvimento econômico e cultura.

\section{Buscando Soluções - Supermercado Mesa Pronta (2001)}

No mesmo ano apostou-se numa outra forma de colocar em discussão nas ondas do rádio as questões ligadas à cidadania. Foi produzida uma série de rádionovelas abordando temas como educação, saúde, trabalho, meio ambiente e direitos do cidadão. São 20 dramatizações desenvolvidas num ambiente de um supermercado, batizado de "Supermercado Mesa Pronta". O objetivo principal deste material é tratar o tema de forma descontraída e próxima ao público ouvinte, estimulando o debate sobre a realidade local. 


\section{Buscando Soluções - Supermercado Mesa Pronta (2003)}

Seu Genésio, Laura, Arthur, Bianca e a população de Campo Verde vivem histórias muito parecidas com a da população brasileira neste volume. São 16 dramatizações, cada uma com cinco minutos de duração, que abordam temas como a saúde da mulher, o racismo, a reciclagem de lixo, dentre outros.

\section{Buscando Soluções - Sempre Uma Boa Idéia no Ar (2003)}

Este volume proporciona aos ouvintes viajar de Roraima ao Rio Grande do Sul e conhecer idéias inovadoras para a promoção da educação, dos direitos humanos, da participação popular, dentre outros temas abordados

\section{Buscando Soluções - Supermercado Mesa Pronta (2004)}

Discutir temas ligados à cidadania com bom humor e criatividade. Esse é o convite que os novos programas Supermercado Mesa Pronta fazem aos ouvintes de todo o Brasil. Na terceira série de radionovelas, os capítulos abordam assuntos como alcoolismo, desemprego, direito dos idosos, agricultura e violência, trabalho escravo e eleições. 


\section{PÁGINAS NA INTERNET}

A página na internet do Programa Gestão Pública e Cidadania oferece informações sobre o ciclo de premiação anual e acesso ao banco de dados, estudos de caso, vídeos, publicações e programas de rádio.

\section{http://inovando.fgvsp.br}

O Programa Gestão Pública e Cidadania desenvolveu também a página na internet do Banco de Dados de Fomento Social, onde é possível identificar instituições de fomento de ações locais de desenvolvimento social e econômico. Você terá acesso a instituições que oferecem recursos a fundo perdido, linhas de crédito, concessões de bolsas, financiamento e auxílio direto na gestão de projetos, além de assessoria para capacitação de pessoal, para fortalecimento institucional ou para transferência e conhecimento técnico.

http://fosocial.fgvsp.br 


\section{FICHA TÉCNICA}

\section{Organizadores:}

Peter Spink

Estêvão Passos Eller

Lilia Asuca Sumiya

Marco Antonio Teixeira

Apresentação:

Peter Spink

\section{Texto Analítico:}

Marco Antonio Teixeira

Estêvão Passos Eller

\section{Textos Informativos e Explicativos:}

Estêvão Passos Eller

Lilia Asuca Sumiya

\section{Revisão Técnica:}

Estêvão Passos Eller

Lilia Asuca Sumiya

Cadastro dos dados dos programas, projetos e atividades:

Maria Fernanda Alessio

Mary Cheng

Janaína Valéria Mattos

Daniela Paschoal Sanches

Fernanda Izidoro

Augusto Marcondes

Daniela Azevedo

Gustavo Amore

Reuel Magno Passos Eller

Patrícia Tiemi Y. Kawahara

Estêvão Passos Eller

Lilia Asuca Sumiya

\section{Revisão dos Dados:}

Daniela Paschoal Sanches

Estêvão Passos Eller

Lilia Asuca Sumiya

\section{Elaboração dos Resumos:}

Estêvão Passos Eller

Janaína Mattos

Maria Fernanda Alessio

Mary Cheng

Melissa Godoy

Lilia Asuca Sumiya

Revisão dos Resumos:

Lilia Asuca Sumiya

Apoio Geral - Ciclo de Premiação 2004:

Daniela Paschoal Sanches

Fabiana P. S. de Moura

Marlei de Oliveira

Rosa Maria de Lima e Silva

\section{Apoio Técnico:}

Cássia Gucoff

\section{Capa}

Carlos André Inácio 